\title{
Visuospatial Memory Alteration in Alzheimer's Disease
}

\author{
Sen $\operatorname{Lin}^{1}$
}

Received: 11 June 2020/Accepted: 19 July 2020/Published online: 2 August 2020

(C) Shanghai Institutes for Biological Sciences, CAS 2020

\begin{abstract}
Although the treatment and drug discovery for Alzheimer's disease $(\mathrm{AD})$ are limited and some have failed recently, the search for potential biomarkers and early predictors continue. $\mathrm{AD}$ is not only characterized by the decline of episodic memory, but also by amnestic mild cognitive impairment (aMCI) that deserves more attention. MCI is a positive prodrome of subsequent $\mathrm{AD}$, and the rate of conversion to $\mathrm{AD}$ can rise to $50 \%$ at $2-3$ years from the initial stage. It has been reported that, after 6 years, 61 out of $76 \mathrm{MCI}$ patients (mean age $=81$ years) converted to $\mathrm{AD}$ $[1,2]$. The assessment of visuospatial abilities, which are necessary pre-requisites of independent mobility in the environment, is crucial to monitor elderly people's wellbeing. Navigational impairment in AD patients has been linked to a disorder of extrastriate visual cortical motion processing that is reflected in specific perceptual and memory measures of spatial abilities [3].
\end{abstract}

Cortical visual processing is divided into distinct dorsal and ventral streams that originate in the striate cortex. The dorsal stream processes spatial or "where/how" information for object localization, while the ventral stream processes visual or "what" features for object recognition $[4,5]$. The dorsal stream actually gives rise to three distinct, major pathways: parieto-prefrontal, parieto-premotor, and parieto-medial temporal lobe (MTL) pathways. A new neural framework for primates proposed by Mishkin and collaborators indicates that the MTL pathway (including the hippocampus, parahippocampal gyrus (PHG), and

Sen Lin

sam.lin@tmmu.edu.cn

1 Department of Ophthalmology, Daping Hospital, Army Medical Center of the People's Liberation Army, Army Medical University, Chongqing 400042, China entorhinal cortex) and the frontal lobes are critical regions for visuospatial memory (Fig. 1); they are key structures supporting both visuospatial and mnemonic processes, responding strongly to visual scenes such as landscapes, cityscapes, and buildings [5-7]. Neuropathology and structural MRI studies have demonstrated that the MTL is the earliest affected brain region in $\mathrm{AD}$ [8]. Although clinical evidence from patients with lesions and research on non-human primates have enriched the anatomy of visuospatial pathways, the detailed neural circuits are still under investigation. The postrhinal cortex (POR), which is homologous to the PHG in humans, has been shown by Zhang's group to include a POR-ventrolateral orbitofrontal cortex (vlOFC) glutamatergic pathway that specifically regulates spatial memory retrieval [9]. The anterior temporal lobe-OFC pathway (including the PHG) is connected by the uncinate fasciculus (UF), which originates within the OFC and then forms a single bundle that projects dorsally and arches down to reach the anterior temporal lobe. Compared with the traditional fMRI and perception research, their refined serial studies dissected the basic neural circuit of the POR-vlOFC pathway, and provided a deeper understanding of UF function and dysfunction related to disorders of memory, as in $\mathrm{MCI}$ and $\operatorname{AD}[9,10]$.

In the current issue of Neuroscience Bulletin, Lin Zhu and collaborators report the relationship between and impairment of the POR-vlOFC circuit and visuospatial memory deficits through retrograde tracing and in vivo local field potential recording in 5XFAD mice as well as the investigation of alterations of the PHG-OFC circuit in AD patients by MRI [10]. They first found impaired integrity of the glutamatergic POR-vlOFC circuit along with the visuospatial memory deficit. In mice, they also used opto-stimulation to successfully improve the impaired visuospatial memory. In addition, by MRI measurement of 


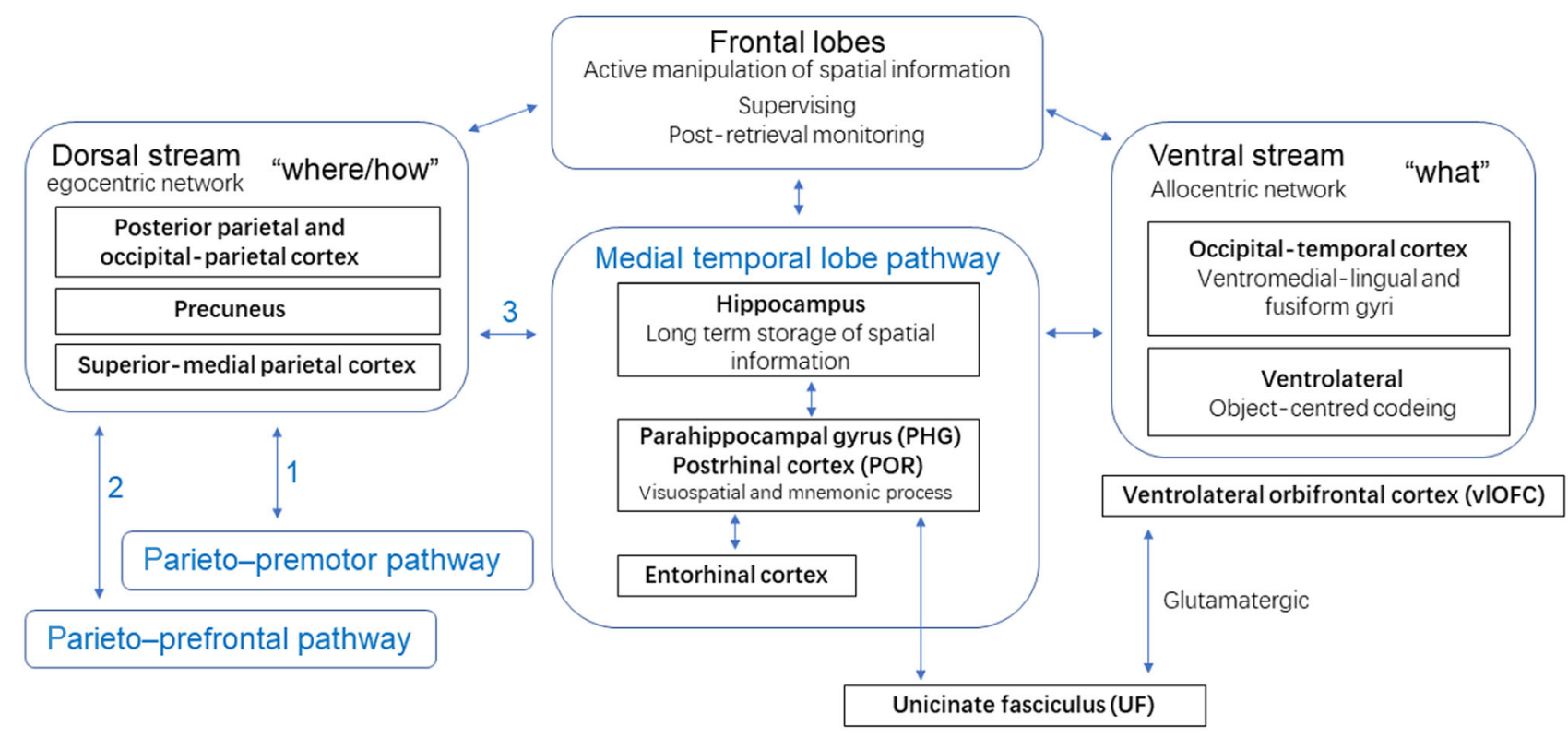

Fig. 1 Graphic illustration of the relationships of neocortical regions and the dorsal and ventral streams. (1) The parieto-premotor pathway: the occipito-parietal circuit is linked to a pre-arcuate region and the caudal portions of the banks of the principal sulcus in the prefrontal cortex. (2) The parieto-prefrontal pathway has major sources in areas of V6A and the medial intraparietal area targeting the dorsal premotor

progressive damage in the PHG-OFC in aMCI converters and non-converters and healthy controls, they found that the annual change of diffuse tensor imaging (DTI) is a clinical estimator to indicate the conversion of aMCI to $\mathrm{AD}$, and may aid the development of methods to treat $\mathrm{AD}$. In addition, their findings also expand the MTL pathway by revealing a glutamatergic POR-vlOFC circuit associated with visuospatial memory, containing the mainly allocentric spatial representation of scenes. Furthermore, the findings illustrate the neuroanatomical basis of visuospatial memory and provide an opportunity to explore new clinical predictors of $\mathrm{AD}$ conversion and novel methods for the early diagnosis of $\mathrm{AD}$.

Comparisons of rodents, non-human primates, and humans need to be distinguished in these initial findings, by experimental and clinical fMRI studies. The neural circuits in human and non-human primates are more complex than in rodents. The diversity of neurons, the specific metabolism in specific brain regions, and the novel neural activity associated with visuospatial memory enrich the understanding of the POR-vlOFC visuospatial pathway.

Acknowledgements This editorial article was supported by the Basic Research and Scientific Frontier Foundation of Chongqing Municipality, China (cstc2019jcyj-msxmX0010). cortex, and the ventral intraprietal area projecting to the ventral premotor cortex, mediating eye movements. (3) The MTL pathway includes portions of the entorhinal cortex, parahippocampal cortex, and perirhinal cortex, receiving spatial information from the posterior cingulate cortex (PCC) and retrosplenial cortex.

\section{References}

1. Petersen RC, Doody R, Kurz A, Mohs RC, Morris JC, Rabins PV, et al. Current concepts in mild cognitive impairment. Arch Neurol 2001, 58: 1985-1992.

2. Morris JC, Storandt M, Miller JP, McKeel DW, Price JL, Rubin $\mathrm{EH}$, et al. Mild cognitive impairment represents early-stage Alzheimer disease. Arch Neurol 2001, 58: 397-405.

3. Iachini I, Iavarone A, Senese VP, Ruotolo F, Ruggiero G. Visuospatial memory in healthy elderly, AD and MCI: a review. Curr Aging Sci 2009, 2: 43-59.

4. Weller RE. Two cortical visual systems in Old World and New World primates. Prog Brain Res 1988, 75: 293-306.

5. Kravitz DJ, Saleem KS, Baker CI, Mishkin M. A new neural framework for visuospatial processing. Nat Rev Neurosci 2011, 12: $217-230$.

6. Epstein R and Kanwisher N. A cortical representation of the local visual environment. Nature 1998, 392: 598-601.

7. Epstein RA and Ward EJ. How reliable are visual context effects in the parahippocampal place area? Cereb Cortex 2010, 20: 294-303.

8. Mosconi L. Brain glucose metabolism in the early and specific diagnosis of Alzheimer's disease. FDG-PET studies in MCI and AD. Eur J Nucl Med Mol Imaging 2005, 32: 486-510.

9. Qi X, Du ZJ, Zhu L, Liu X, Xu H, Zhou Z, et al. The glutamatergic postrhinal cortex-ventrolateral orbitofrontal cortex pathway regulates spatial memory retrieval. Neurosci Bull 2019, 35: 447-460.

10. Zhu L, Wang Z, Du Z, Qi X, Shu H, Liu D, et al. Impaired parahippocampal gyrus-orbitofrontal cortex circuit associated with visuospatial memory deficit as a potential biomarker and interventional approach for Alzheimer disease. Neurosci Bull 2020. https://doi.org/10.1007/s12264-020-00498-3. 